\title{
A novel cognitive radio spectrum assignment scheme for maximizing system utilization
}

\author{
Jung-Chieh Chen ${ }^{\text {a) }}$ \\ Department of Optoelectronics and Communication Engineering, \\ National Kaohsiung Normal University, Kaohsiung 802, Taiwan \\ a)jcchen@m.ieice.org
}

\begin{abstract}
This paper deals with the problem of spectrum sharing among primary and secondary users in a cognitive radio. To use the spectrum efficiently and flexibly, the cognitive radio spectrum allocation problem was formulated as a graph coloring problem (GCP). However, GCP is known to be a nondeterministic polynomial-time (NP)hard and cannot be solved for the practical system. Inspired by the efficiency of the cross-entropy (CE) method for finding near-optimal solutions in huge search spaces, this paper proposes the application of the $\mathrm{CE}$ method to allocate cognitive radio spectrum to achieve nearoptimal spectrum utilization. Simulation results show that the average reward values obtained using the proposed CE method are better than those obtained using the conventional method.
\end{abstract}

Keywords: cross-entropy method, cognitive radio, spectrum allocation

Classification: Science and engineering for electronics

\section{References}

[1] W. Zhang, J. Yang, Q. Yan, and L. Zhang, "Optimal multiband spectrum sensing in cognitive radio," IEICE Electron. Express, vol. 7, no. 20, pp. 1557-1563, Oct. 2010.

[2] S. Haykin, "Cognitive radio: brain-empowered wireless communications," IEEE J. Sel. Areas Commun., vol. 23, no. 2, pp. 201-220, Feb. 2005.

[3] I. F. Akyildiz, W. Lee, M. C. Vuran, and S. Mohanty, "Next generation/dynamic spectrum access/cognitive radio wireless networks: a survey," Comput. Netw., vol. 50, no. 13, pp. 2127-2159, Sept. 2006.

[4] C. Peng, H. Zheng, and B. Y. Zhao, "Utilization and fairness in spectrum assignment for opportunistic spectrum access," ACM Mobile Netw. Appl., vol. 11, no. 4, pp. 555-576, May 2006.

[5] Z. Zhao, Z. Peng, S. Zheng, and J. Shang, "Cognitive radio spectrum allocation using evolutionary algorithms," IEEE Trans. Wireless Commun., vol. 8, no. 9, pp. 4421-4425, Sept. 2009.

[6] C. Liao, J. Chen, Y. Tang, and S. Li, "Parallel algorithm of spectrum allocation in cognitive radio," J. Electron. Inf. Technol., vol. 29, no. 7, pp. 1608-1611, July 2007. 
[7] R. Y. Rubinstein and D. P. Kroese, The Cross-Entropy Method., Berlin, Germany: Springer, 2004.

[8] J.-C. Chen and C.-K. Wen, "A novel cognitive radio adaptation for wireless multicarrier systems," IEEE Commun. Lett., vol. 14, no. 7, pp. 629631, July 2010.

[9] Y. Zhang, C. Ji, W. Malik, D. O'Brien, and D. Edwards, "Receive antenna selection for MIMO systems over correlated fading channels," IEEE Trans. Wireless Commun., vol. 8, no. 9, pp. 4393-4399, Sept. 2009.

\section{Introduction}

With increasing demands for the use of wireless radio spectrum, cognitive radio has attracted considerable attention in recent years due to its desirable properties, such as spectrum sensing [1], spectrum management, spectrum mobility, and spectrum sharing $[2,3]$. To fully utilize the scarce spectrum resources efficiently and flexibly, a cognitive radio allows unlicensed users (secondary users) to access unused spectrum bands dynamically without interfering with the licensed users (primary users), thereby improving spectrum efficiency. The question now is how to deal with the problem of spectrum sharing among primary and secondary users to maximize spectrum utilization of cognitive radios.

To handle the problem of spectrum sharing in cognitive radios, various approaches were proposed $[4,5,6]$. Among those proposed, the particle swarm optimization (PSO)-based spectrum allocation algorithm performs better than the other schemes. However, the performance of the PSO-assisted spectrum allocation algorithm still has room for improvement. Inspired by the success of the cross-entropy (CE) method [7] in the implementation of cognitive engine for wireless multicarrier transceivers in our previous work [8], this paper proposes the application of the $\mathrm{CE}$ method to allocate the available channels to unlicensed users to maximize the system utilization in cognitive radios. Simulation results demonstrate that the proposed CE-assisted spectrum allocation algorithm outperforms the PSO-assisted spectrum allocation algorithm.

\section{System Model and Problem Definition}

The spectrum allocation model and assumption of this paper basically follows $[4,5]$. We first define the channel availability matrix, an $N \times M$ binary matrix $\mathbf{H}=\left[h_{n, m}\right]_{N \times M}$, where we assume that there are $N$ secondary users competing for $M$ orthogonal spectrum channel in a cognitive radio. The $(n, m)^{t h}$ entry of matrix $\mathbf{H}$ can then be expressed as

$$
h_{n, m}=\left\{\begin{array}{ll}
1, & \text { if channel } m \text { is available to user } n \\
0, & \text { otherwise }
\end{array} .\right.
$$

Depending on a user's location or environment, the bandwidth/ throughput achieved by different bands is generally different. Thus, to de- 
scribe the reward of user $n$ for using channel $m$ for communication, we next define the channel reward matrix, an $N \times M$ matrix $\mathbf{B}=\left[b_{n, m}\right]_{N \times M}$, where $b_{n, m}$ characterizes the reward when a secondary user $n$ adopts channel $m$.

When users $n$ and $k$ use the same channel $m$ simultaneously, they would interfere with each other. To avoid interference, we define the interference constraint matrix, an $N \times N \times M$ binary matrix $\mathbf{C}=\left[c_{n, k, m}\right]_{N \times N \times M}$. The $(n, k, m)^{t h}$ entry of matrix $\mathbf{C}$ can then be expressed as

$$
c_{n, k, m}= \begin{cases}1, & \text { if users } n \text { and } k \text { use channel } m \text { simultaneously } \\ 0, & \text { otherwise }\end{cases}
$$

Note that the condition " $c_{n, k, m}=1$ " implies that user $n$ will interfere user $k$ if they both use channel $m$. On the other hand, $h_{n, m}$ means that channel $m$ is available for secondary user $n$ to use. Therefore, if $c_{n, k, m}=1, h_{n, m}$ must be equal to 1 .

Finally, a conflict-free channel assignment matrix, an $N \times M$ binary matrix $\boldsymbol{\omega}=\left[\varpi_{n, m}\right]_{N \times M}$, represents a solution to spectrum-sharing problems and describes which channel is assigned to which secondary user. The $(n, m)^{t h}$ entry of matrix $\boldsymbol{\omega}$ can then be expressed as

$$
\varpi_{n, m}=\left\{\begin{array}{ll}
1, & \text { if channel } m \text { is allocated to secondary user } n \\
0, & \text { otherwise }
\end{array} .\right.
$$

It is important to emphasize that an assignment $\boldsymbol{\omega}$ is conflict-free if a secondary user is only assigned with channels which do not conflict with any other user. For this reason, $\boldsymbol{\omega}$ has to satisfy all the constraints defined by $\mathbf{C}$, which can be described by

$$
\varpi_{n, m}+\varpi_{k, m} \leq 1 \quad \text { if } c_{n, k, m}=1, \quad \forall 1 \leq n, k \leq N, \quad 1 \leq m \leq M .
$$

For ease of presentation, we use $\Lambda_{\mathbf{H}, \mathbf{C}}$ to denote the set of valid spectrum assignments for a given $\mathbf{H}$ and $\mathbf{C}$.

With the notation above, the goal of this paper is to maximize total spectrum utilization in the system. Consequently, spectrum allocation problem can be defined as the following optimization problem

$$
\boldsymbol{\omega}^{\star}=\arg \max _{\boldsymbol{\omega} \in \Lambda_{\mathbf{H}, \mathbf{C}}} \mathcal{R}(\boldsymbol{\omega})
$$

where,

$$
\mathcal{R}(\boldsymbol{\omega})=\sum_{n=1}^{N} \sum_{m=1}^{M} \varpi_{n, m} \cdot b_{m, n} .
$$

Here, $\boldsymbol{\omega}^{\star}$ denotes the global optimal conflict-free channel assignment matrix and $\mathcal{R}(\boldsymbol{\omega})$ represents the total spectrum utilization in the system for a given conflict-free channel assignment $\boldsymbol{\omega}$.

\section{The CE-based Spectrum Allocation Method}

CE was first proposed by Rubinstein [7] to solve rare event probability estimations and was soon successfully applied to solve combinatorial optimization 
problems by considering an optimal event as a rare event, such as nondeterministic polynomial-time (NP) hard problems. This section focuses only on the application of $\mathrm{CE}$ to the optimization problem being considered in (5). Refer to [7] for more technical details on the method.

To proceed with $\mathrm{CE}$, the first step is to transform the deterministic optimization problem (5) into a family of stochastic sampling problems. As in typical CE applications for discrete optimization problems, we can define a family of Bernoulli probability mass functions associated with the channel assignment matrix $\boldsymbol{\omega}=\left[\varpi_{n, m}\right]_{N \times M}, \varpi_{n, m} \in\{0,1\}$, given by

$$
f(\boldsymbol{\omega} ; \mathbf{p})=\prod_{n=1}^{N} \prod_{m=1}^{M} p_{n, m}^{1_{n, m}(\boldsymbol{\omega})}\left(1-p_{n, m}\right)^{1-1_{n, m}(\boldsymbol{\omega})}
$$

where the indicator function, $1_{n, m}(\boldsymbol{\omega}) \in\{0,1\}$, indicates whether the $(n, m)^{t h}$ element of $\boldsymbol{\omega}$ is selected. If channel $m$ is allocated to secondary user $n$, $1_{n, m}(\boldsymbol{\omega})$ is set to 1 . Each element of $\boldsymbol{\omega}$ is modeled as an independent Bernoulli random variable with probability mass function $\operatorname{Pr}\left[\varpi_{n, m}=1\right]=$ $p_{n, m}, \operatorname{Pr}\left[\varpi_{n, m}=0\right]=1-p_{n, m}$, for $n=1,2, \ldots, N$ and $m=1,2, \ldots, M$.

Formally, the proposed CE-assisted spectrum allocation algorithm can be described using the following two-phase iterative steps. At iteration $l, N_{s}$ samples $\left\{\boldsymbol{\omega}^{(i, l)}\right\}_{i=1}^{N_{s}}$ are generated according to a certain known distribution $f\left(\cdot ; \mathbf{p}^{(l-1)}\right)$. Then, the new proposal $f\left(\cdot ; \mathbf{p}^{(l)}\right)$ is updated to close the target distribution $f\left(\cdot ; \mathbf{p}^{\star}\right)$ by minimizing the Kullback-Leibler divergence, that is, cross-entropy. After a number of iterations, the generated samples are equal to or close to being equal to $\boldsymbol{\omega}^{\star}$. The proposed algorithm is described in detail below.

- Step 1: Set the iteration counter $l:=1$ and initialize the probability matrix $\mathbf{p}^{(0)}=\left[p_{n, m}^{(0)}\right]_{N \times M}$ with $p_{n, m}^{(0)}=\frac{1}{2}$ for $n=1,2, \ldots, N$ and $m=1,2, \ldots, M$.

- Step 2: Generate $N_{s}$ random samples $\left\{\boldsymbol{\omega}^{(i, l)}\right\}_{i=1}^{N_{s}}$ from the density function $f\left(\cdot ; \mathbf{p}^{(l-1)}\right)$.

- Step 3: Apply the projection method in [9] to ensure that each sample $\boldsymbol{\omega}^{(i, l)}$ meets the constraint of valid spectrum assignments, i.e., $\boldsymbol{\omega}^{(i, l)} \in$ $\Lambda_{\mathbf{H}, \mathbf{C}}$.

- Step 4: Calculate the performance functions $\left\{\mathcal{R}\left(\boldsymbol{\omega}^{(i, l)}\right)\right\}_{i=1}^{N_{s}}$ and determine the elite set using $\Omega^{(l)}=\left\{\boldsymbol{\omega}^{(i, l)}: \mathcal{R}\left(\boldsymbol{\omega}^{(i, l)}\right) \geq \gamma^{(l)}\right\}$, where $\gamma^{(l)}$ is the $(1-\rho)$ sample quantile of the performances at iteration $l$.

- Step 5: Update parameter $\mathbf{p}^{(l)}=\left[p_{n, m}^{(l)}\right]_{N \times M}$ according to

$$
p_{n, m}^{(l)}=\frac{\sum_{i=1}^{N_{s}} I_{\left\{i \in \Omega^{(l)}\right\}} 1_{n, m}\left(\boldsymbol{\omega}^{(i, l)}\right)}{\left.\sum_{i=1}^{N_{s}} I_{\left\{i \in \Omega^{(l)}\right.}\right\}},
$$


where $I_{\left\{i \in \Omega^{(l)}\right\}}$ is an indicated variable defined by

$$
I_{\left\{i \in \Omega^{(l)}\right\}}=\left\{\begin{array}{ll}
1, & \text { if } i \in \Omega^{(l)} \\
0, & \text { otherwise }
\end{array} .\right.
$$

- Step 6: Smooth parameter $\mathbf{p}^{(l)}$ via

$$
\mathbf{p}^{(l)}=\lambda \times \mathbf{p}^{(l)}+(1-\lambda) \times \mathbf{p}^{(l-1)},
$$

where $\lambda$ is the smoothing parameter, with $0<\lambda \leq 1$.

- Step 7: If the stopping criterion is not reached, then set $l:=l+1$. Go back to Step 2. The stopping criterion is the predefined number of iterations.

\section{Experimental Results and Discussion}

We simulated a cognitive radio system with $N=30$ and $M=50$, where $\mathbf{H}$ and $\mathbf{C}$ are random binary matrices, and $\mathbf{B}$ is an integer matrix whose entries are natural numbers randomly generated from $[0, N]^{1}$. To verify the robustness of the proposed method, we tested the proposed CE algorithm using three different unused rates for $\mathbf{H}$, where the unused rate, $\eta$, is defined as the percentage of the total number of channels available to secondary users. For comparison, the PSO-based spectrum allocation algorithm in [5] was tested. Note that PSO and CE are population-based search methods. The total number of evaluations for PSO and CE is given by multiplying the number of generations $(l)$ by the sample size $\left(N_{s}\right)$.

The average reward values versus the total number of evaluations for three unused rates, $\eta=40 \%, \eta=50 \%$, and $\eta=60 \%$, using PSO and $\mathrm{CE}$, are shown in Fig. 1, where the average reward values are obtained by averaging over 100 independent trials. In addition, the average reward when the algorithms converge to the desired solutions is also shown in Table I, where convergence is defined as the point at which the reward value is no longer improved. Based on the simulation results, the following observations were made. First, as expected, the average reward values improved with an increase in the total number of evaluations for both the PSO-assisted and the proposed CE-assisted spectrum allocation algorithms. Second, the highest average reward values achieved by the proposed $\mathrm{CE}$ were superior compared with those by PSO, regardless of the unused rate settings employed. Third, the convergence speed of the proposed $\mathrm{CE}$ algorithm was lower than that of the PSO algorithm at the initial stage. However, after the initial stage, the convergence speed of the proposed CE algorithm improved greatly until

\footnotetext{
${ }^{1}$ In [4], the reward that the secondary user $n$ gets for using channel $m$ is set to $b_{n, m}=$ $d_{n, m}^{2}$, where $d_{n, m}$ is the interference range of user $n$ with channel $m$. This setup means that the reward is proportional to the service coverage area. In simulation, $d_{n, m}$ can be determined by randomly assigning the locations of the primary and secondary users in the given area. However, for ease of simulation, an alternative approach for generating $b_{n, m}$ is to randomly choose a natural number from $[0, N]$, as was done in [6]. In this paper, we adopt the later approach because of its simplicity. 


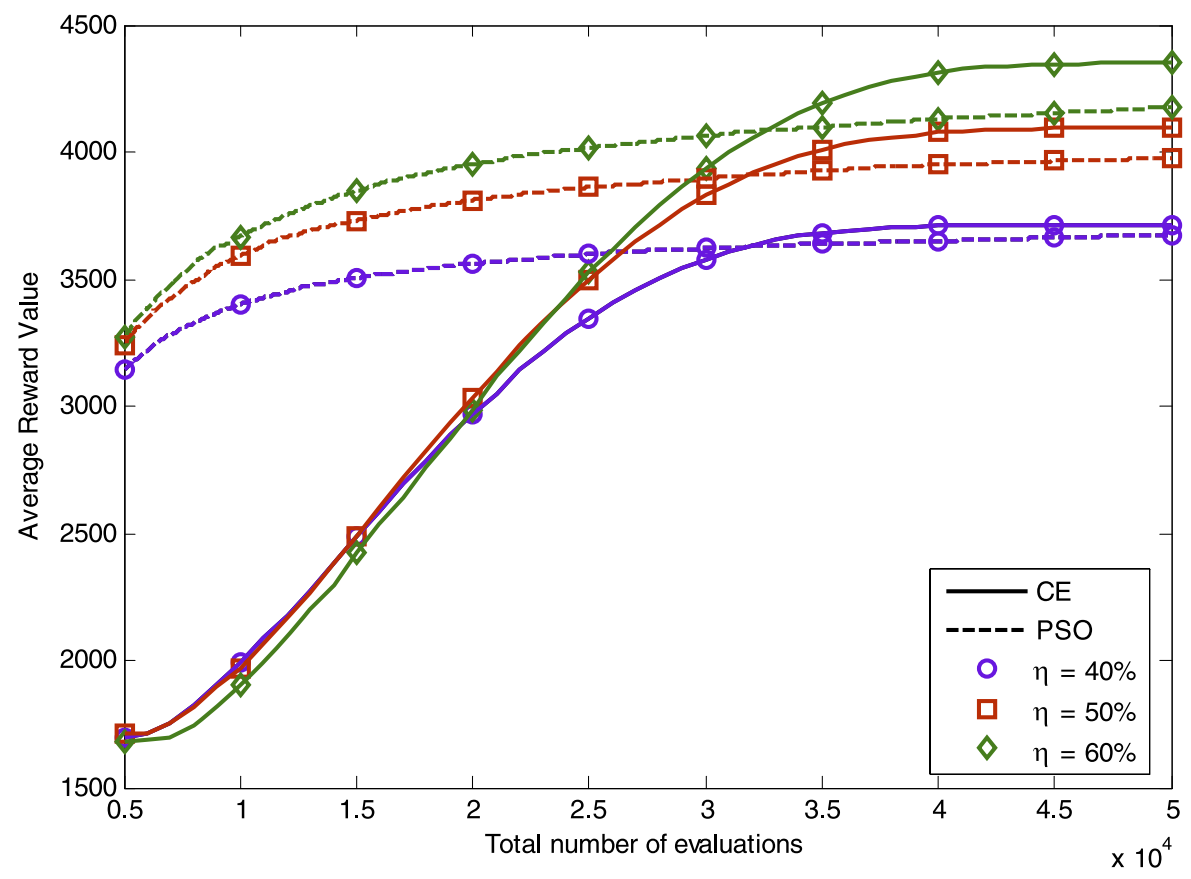

Fig. 1. Average rewards: PSO vs. CE.

Table I. Unused rate $(\eta)$ versus the average rewards for PSO and CE.

\begin{tabular}{|c|c|c|c|}
\hline & $\eta=40 \%$ & $\eta=50 \%$ & $\eta=60 \%$ \\
\hline PSO & 3669.7 & 3964.7 & 4157.4 \\
\hline CE & 3705.2 & 4060.4 & 4358.5 \\
\hline
\end{tabular}

it reached the near-optimal solution. On the other hand, the PSO-assisted spectrum allocation method was easily trapped in local solutions, leading to slow convergence after the initial stage.

\section{Conclusion}

This paper presented a CE-based method that was used to search a nearly optimal spectrum allocation for a cognitive radio network to improve spectrum utilization. Simulation results showed that the proposed method outperforms the PSO-assisted spectrum allocation method in terms of the highest average reward values, regardless of the unused rate settings employed.

\section{Acknowledgments}

This work was supported in part by the National Science Council, Taiwan, 2011. 